\title{
Seismic Evidence for Olivine Phase Changes at the 410- and 660-Kilometer Discontinuities
}

\author{
Sergei Lebedev, ${ }^{1 *}$ Sébastien Chevrot, ${ }^{2}$ Rob D. van der Hilst ${ }^{1}$
}

The view that the seismic discontinuities bounding the mantle transition zone at 410- and 660-kilometer depths are caused by isochemical phase transformations of the olivine structure is debated. Combining converted-wave measurements in East Asia and Australia with seismic velocities from regional tomography studies, we observe a correlation of the thickness of, and wavespeed variations within, the transition zone that is consistent with olivine structural transformations. Moreover, the seismologically inferred Clapeyron slopes are in agreement with the mineralogical Clapeyron slopes of the $(\mathrm{Mg}, \mathrm{Fe})_{2} \mathrm{SiO}_{4}$ spinel and postspinel transformations.

The seismic velocity discontinuities at global average depths of 410 and $660 \mathrm{~km}$ have been attributed to isochemical phase transformations, from olivine to wadsleyite $(\alpha \rightarrow \beta)$ and from spinel to perovskite and magnesiowüstite $(\gamma \rightarrow p v+m w)$, respectively (1-9). However, some recent in situ studies of the postspinel transformation suggested that the $660-\mathrm{km}$ discontinuity may instead be due to the transformation of majorite garnet $(g t)$ to perovskite in a pyroxene-garnet-dominant transition zone (TZ) $(10,11)$. Tests of the seismic predictions from the olivine model have yielded ambiguous results, including a surprisingly weak correlation between the thickness of $\left(H_{\mathrm{TZ}}\right)$ and seismic velocities within the TZ $(12,13)$. Here, we show that the patterns of $S$-velocity heterogeneity and discontinuity topography in the East AsianAustralian TZ are consistent with olivine transitions as the prime cause for the 410 - and 660-km discontinuities (410 and 660).

The Clapeyron slopes (of the phase boundaries in $P-T$ space) of the $\alpha \rightarrow \beta$ and $\gamma \rightarrow p v+m w$ reactions are probably positive and negative, respectively (14). If the seismically inferred Clapeyron slopes $\gamma_{410}$ and $\gamma_{660}$ are similar (15), the 410 and 660 should be deflected toward (away from) each other at relatively high (low) temperatures. $H_{\mathrm{TZ}}$ would then correlate with temperature and temperature-dependent seismic velocities within the TZ (Fig. 1A). If, instead, the 660 is caused by the $g t \rightarrow p v$ transition, which has a positive Clapeyron slope (16), the correlation between $H_{\mathrm{TZ}}$ and the seismic velocities would be weak or absent. Pertinent seismic

${ }^{1}$ Department of Earth, Atmospheric, and Planetary Sciences, Room 54-512, Massachusetts Institute of Technology, Cambridge, MA 02139, USA. 'Observatoire Midi Pyrénées, 14 Avenue Edouard Belin, Toulouse, France.

*To whom correspondence should be addressed. Email: sergei@quake.mit.edu evidence has so far been equivocal (17).

We use converted-wave $(P \mathrm{~d} s)$ delay-time measurements along with $S$-velocity values in the $\mathrm{TZ}\left(V_{\mathrm{S}}^{\mathrm{TZ}}\right)$ estimated from tomographic models of East Asia (18) and Australia (19), which were computed with large sets of vertical-component seismograms. We measured
$t_{P 660 s}$ and $t_{P 410 s}$ arrival times from stacks of rotated three-component seismograms, deconvolved by the principal component of the recorded $P$-wave train $(12,20)$, and obtained $t_{\text {diff }}=t_{p 660 s}-t_{p 410 s}$ values with "bootstrap" errors (21) for 12 stations in East Asia and Australia. We rejected traces with a low signal-to-noise ratio, stacks that did not show both $P 660 s$ and $P 410$ s, and stations for which the number of the accepted traces was insufficient to determine $t_{P 660 s}, t_{P 410 s}$, and their uncertainties (22). Because the paths of $P 660$ s and $P 410 s$ are essentially the same below 660 and above 410 (Fig. 1B), to firstorder, $t_{\text {diff }}$ depends only on the thickness of and seismic velocities within the TZ. The $t_{\text {diff }}$ measurements represent lateral averages over $\approx 500 \mathrm{~km}(23)$.

In the tomographic models, the upper 200 to $300 \mathrm{~km}$ is constrained primarily by longand intermediate-period Rayleigh waves and deeper structure, including that in the TZ, by thousands of $S$ and multiple- $S$ waveforms. Dense data coverage (Fig. 2) provides 500- to $600-\mathrm{km}$ lateral resolution in the TZ (Fig. 3) $(18,19)$. However, tomographic imaging may underestimate the amplitude of $\mathrm{TZ}$
A

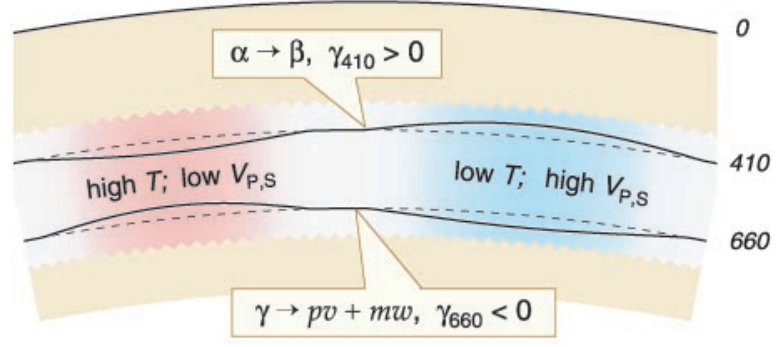

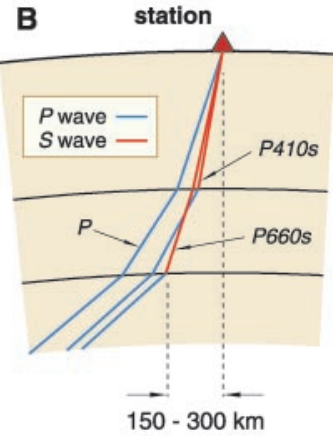

$150-300 \mathrm{~km}$

Fig. 1. (A) Schematic depiction of the transition zone in an olivine-dominant mantle. The $\alpha \rightarrow \beta$ and $\gamma \rightarrow p v+m w$ phase transformations give rise to the 410- and 660-km discontinuities (1-9), and the effective Clapeyron slopes $\gamma_{410}$ and $\gamma_{660}$ have opposite signs. Absent lateral variations in composition, relatively low temperatures $(T)$ cause thickening of the TZ and increase in seismic velocities $\left(V_{P}, V_{S}\right)$; high temperatures cause thinning of the TZ and decrease in $V_{P, S}$ (B) Schematic ray diagram of the $P, P 410$ s, and P660s phases.

Fig. 2. Ray-path coverage used in the $S$-velocity tomography of East Asia (A) (18) and Australia (B) (19). The tomographic models (Fig. 3) are constrained by partitioned waveform inversion (PWI) (38) and automated PWI (18) of multimode Rayleigh waves. Higher modesforming the $S$ and multiple $S$ waves-are sensitive to transition-zone structure. The stations with $t_{\text {diff }}$ measurements are shown with diamonds. Longitude (horizontal axis) is in degrees east, latitude (vertical) is in degrees north (positive) and south (negative).
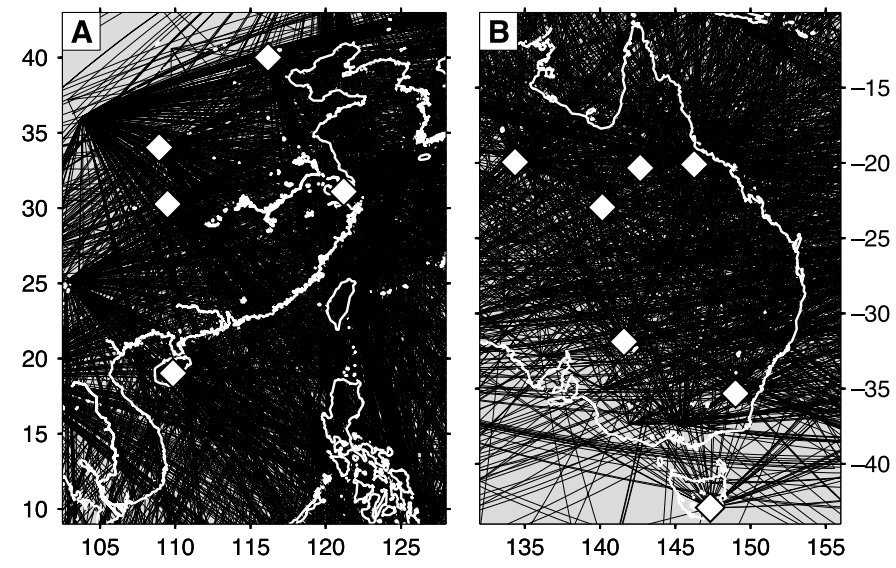


\section{R E P O R T S}

anomalies, and in poorly sampled regions, the retrieved anomalies may be in error due to unresolved trade-offs with structure elsewhere in the model. We therefore did not take the models at face value but used a series of resolution tests (fig. S1) (24) to estimate $V_{\mathrm{S}}$ in the TZ beneath the stations that yielded the $t_{\text {diff }}$ measurements. Reliable estimates for the upper $\left(\delta V_{\mathrm{S}}^{410}\right)$ and lower $\left(\delta V_{\mathrm{S}}^{660}\right)$ parts of the $\mathrm{TZ}$ could be obtained for eight stations (plotted in Fig. 3), with estimated uncertainties ranging from 30 to $60 \mathrm{~m} / \mathrm{s}\left(\delta V_{\mathrm{S}}^{410}\right)$ and 40 to relative to the reference model iasp 91 (25) for a reference period of $1 \mathrm{~s}$, assuming a quality factor $Q=143$ (26).

The required four independent parameters $\left(t_{P 410 s}, t_{P 660 s}, \delta V_{\mathrm{S}}^{410}, \delta V_{\mathrm{S}}^{660}\right)$ could be determined for eight stations only. At these stations, $t_{\text {diff }}$ correlates $(r=0.94)$ with $\delta V_{\mathrm{S}}^{\mathrm{TZ}}=$ $\left(\delta V_{\mathrm{S}}^{410}+\delta V_{\mathrm{S}}^{660}\right) / 2$ (Fig. 4A). The best-fitting line was determined by bivariate regression and has a slope of $0.012 \pm 0.006 \mathrm{~s} /(\mathrm{m} / \mathrm{s})$. For a TZ of constant thickness the differential time $t_{\text {diff }}$ would be smaller in high-velocity regions and larger in low-velocity ones. We $80 \mathrm{~m} / \mathrm{s}\left(\delta V_{\mathrm{S}}^{660}\right)$. We recomputed $\delta V_{\mathrm{S}}^{410,660}$

observe the opposite, implying that $H_{\mathrm{TZ}}$ varies in concert with seismic velocities.

Next, we convert $\delta t_{\text {diff }}$ to transition-zone thickness anomaly $\delta H_{\mathrm{TZ}}$ [using the reference $P$ and $S$ velocities $\left(V_{\mathrm{P}, \mathrm{S}}\right)$ from iasp 91 , our $\delta V_{\mathrm{S}}^{\mathrm{TZ}}$, and assuming $R=\delta \ln V_{\mathrm{S}} / \delta \ln V_{\mathrm{P}}=$ $1.7 \pm 0.7]$ and $\delta V_{\mathrm{S}}^{\mathrm{TZ}}$ to temperature variation $\delta T_{\mathrm{TZ}}$ [using $\partial \ln V_{\mathrm{S}} / \partial T=-1.35 \times 10^{-4} \mathrm{~K}^{-1}$ (27) and a $0.4 \times 10^{-4} \mathrm{~K}^{-1}$ uncertainty of the derivative]. $H_{\mathrm{TZ}}$ correlates with $T_{\mathrm{TZ}}(r=$ 0.98 ), and the slope of the best-fitting line, $-0.13 \pm 0.07 \mathrm{~km} / \mathrm{K}$, is consistent with the $-0.13 \mathrm{~km} / \mathrm{K}$ inferred from the mineralogical Clapeyron slopes of the $\alpha \rightarrow \beta$ and $\gamma \rightarrow p v+$ $m w$ transformations (14) (Fig. 4B).

Encouraged by this result, we use the estimates for $\delta V_{\mathrm{S}}^{410}$ and $\delta V_{\mathrm{S}}^{660}$ and compute the effective Clapeyron slopes $\gamma_{410}$ and $\gamma_{660}$ directly. Expressing the temperature anomalies $\delta T_{410}$ and $\delta T_{660}$ as functions of $\delta V_{\mathrm{S}}^{410}$ and $\delta V_{\mathrm{S}}^{660}$, we obtain one linear equation for each station

$$
\begin{aligned}
\delta H_{\mathrm{TZ}} & =\left(\frac{\partial d}{\partial P}\right)_{660} \cdot \gamma_{660} \cdot \frac{\partial T}{\partial \ln V_{\mathrm{S}}} \cdot \delta \ln V_{\mathrm{S}}^{660} \\
& -\left(\frac{\partial d}{\partial P}\right)_{410} \cdot \gamma_{410} \cdot \frac{\partial T}{\partial \ln V_{\mathrm{S}}} \cdot \delta \ln V_{\mathrm{S}}^{410}
\end{aligned}
$$

Fig. 3. Differential-time $\left(t_{\text {diff }}\right)$ measurements at eight stations superimposed on the tomographic images of the East Asian (A) and Australian (B) transition zones. $V_{\mathrm{s}}$-anomaly values are averaged over the TZ depth range. The Asian model (18) was computed with $400-\mathrm{km}$ a priori smoothing; the Australian model (19) is smoothed over $400 \mathrm{~km}$ a posteriori. Reference $V_{\mathrm{s}}$ values are 5291 and $5311 \mathrm{~m} / \mathrm{s}$ for Asia and Australia, respectively; reference $t_{\text {diff }}$ is $23.9 \mathrm{~s}$ (25).
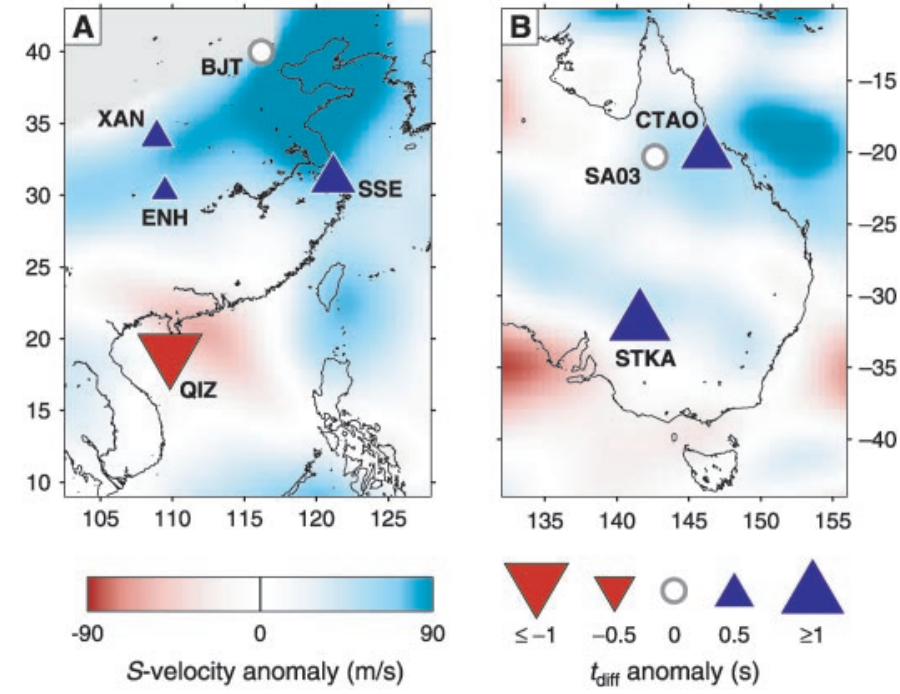

Fig. 4. The correlation between (A) $S$ velocity in the transition zone and the differential time $t_{\text {diff }}$ and between (B) the inferred temperature of the transition zone $T_{\mathrm{TZ}}$ and its thickness $H_{\text {TZ }}$. Seismic velocity anomalies $\delta V_{\mathrm{S}}^{\mathrm{TZ}}$ are vertical averages over the transitionzone depth range, and so are the estimated temperature anomalies $\delta T_{\mathrm{TZ}}$. The data from the East Asian stations are shown with dark-shaded symbols (BJT, with a square; ENH, triangle; QIZ, inverted triangle; SSE, diamond; XAN, circle). The data from the Australian
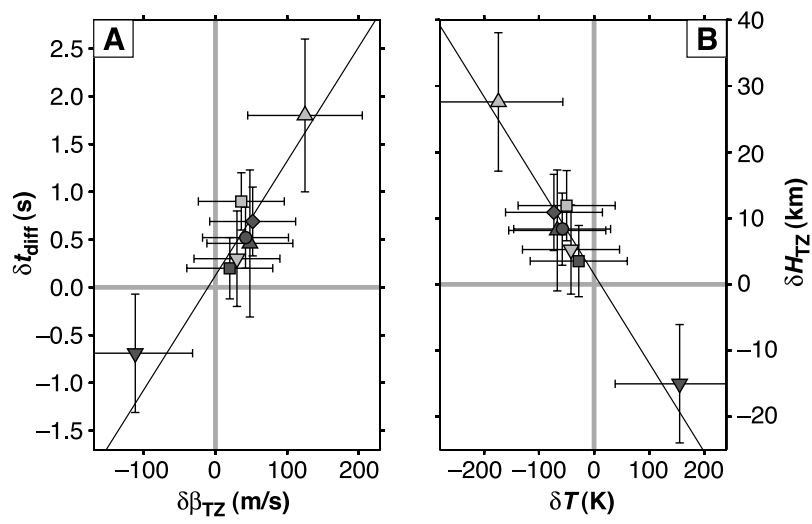

stations are shown with light-shaded symbols (CTAO, with a square; SAO3, inverted triangle; STKA, triangle). where $(\partial d / \partial P)_{410(660)}$ describes the depthpressure relationship at the 410(660). We solve Eq. 1 for $\gamma_{410}$ and $\gamma_{660}$ by minimizing the chi-square (28) function

$$
\begin{aligned}
\chi^{2}= & \sum_{i=1}^{8} \\
& \left(\frac{\delta H_{\mathrm{TZ}}^{i}-\delta H_{\mathrm{TZ}}\left(\gamma_{410}, \gamma_{660} ;\left(V_{\mathrm{S}}^{410}\right)_{\mathrm{i}},\left(V_{\mathrm{S}}^{660}\right)_{i}\right)}{\sigma_{i}}\right)^{2}
\end{aligned}
$$
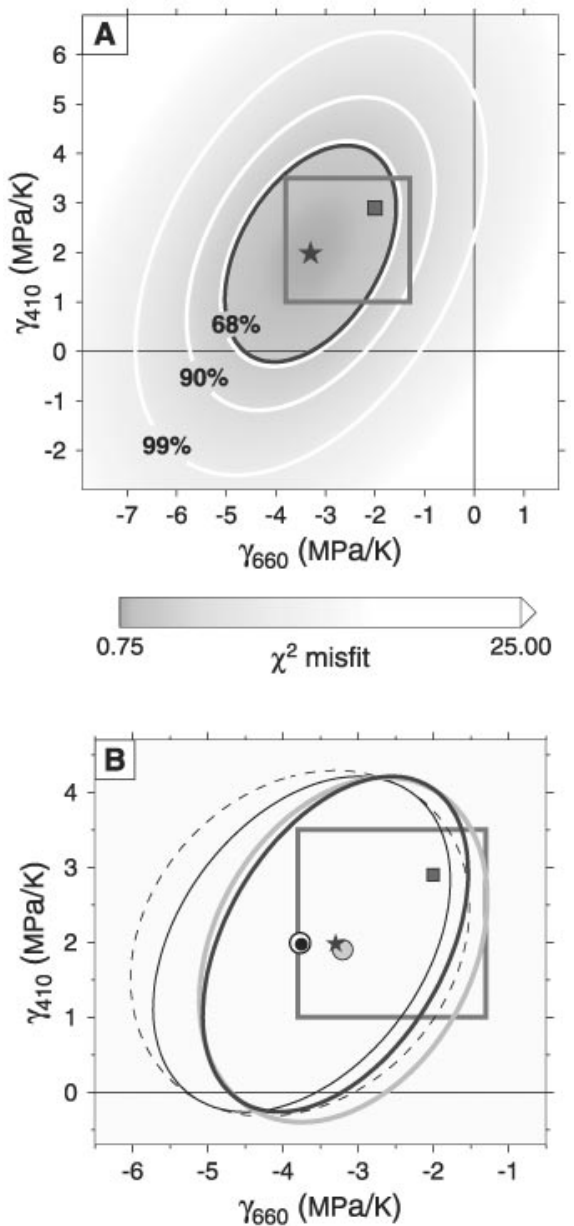

Fig. 5. (A) The effective Clapeyron slopes at the 410- and 660-km discontinuities. $\chi^{2}$ misfit in the $\gamma_{410}-\gamma_{660}$ plane is plotted in the region around the best-fit solution $(\star)$. The values of the mineralogical Clapeyron slopes of $\alpha \rightarrow \beta$ and $\gamma \rightarrow p v+$ $m w$ from (14) [small solid square at $(-2.0 \mathrm{MPa}$; $2.9 \mathrm{MPa})$ ] and the range of measured values from the literature as compiled in (14) (large rectangle) are superimposed as $\gamma_{410}$ and $\gamma_{660}$. (B) The measured effective Clapeyron slopes (A) do not depend strongly on the measurements at the two stations with largest anomalies (Fig. 4). The star and dark-shaded curve denote the best-fit value and $1 \sigma$ error ellipse computed with the complete data set, as in (A). The black and gray circles and lines show the solution of Eq. 1 with one of the two stations (equations) excluded; empty circle and dashed line is the solution with both of the stations excluded. 
where $\delta H_{\mathrm{TZ}}{ }^{i},\left(V_{\mathrm{S}}^{410}\right)_{i}$, and $\left(V_{\mathrm{S}}^{660}\right)_{i}$ are the data at the $i$-th station $(i=1, \ldots, 8)$ and $\delta H_{\mathrm{TZ}}(\ldots)$ is a function of the variables $\gamma_{410}$ and $\gamma_{660}$. The cumulative errors $\sigma_{i}$ are computed for $\delta H_{\mathrm{TZ}}^{i}$ and account for uncertainties of $t_{\text {diff }}^{i}\left(V_{\mathrm{S}}^{410,660}\right)_{i}$, $R$, and $\partial \ln V_{\mathrm{S}} / \partial T$. The solution (Fig. 5A) is consistent with the mineralogic Clapeyron slopes of the olivine transformations (14). The width of the error ellipses accounts for our measurement uncertainties as well as for possible lateral variations in $R( \pm 40 \%)$ and $\delta \ln V_{\mathrm{S}} / \delta T$ $( \pm 30 \%)$. Unlike the slope of the straight line in Fig. 4B, the solution of Eq. 1 is not sensitive to the two extremal data points; excluding either or both results in a small displacement of the best-fit point and a slight widening of the $1 \sigma$ ellipses (Fig. 5B).

The correlation between $t_{\text {diff }}$ and $V_{\mathrm{S}}^{\mathrm{TZ}}$ (and thus between the TZ thickness and temperature) in East Asia-Australia (Fig. 4) contrasts the weak correlation inferred from global $t_{\text {diff }}$ data sets and tomographic models $(12,13,29)$. We suggest that this inconsistency is due to differences in spatial resolution of $t_{\text {diff }}$ measurements, on the one hand, and of $V_{\mathrm{P}}^{\mathrm{TZ}}$ or $V_{\mathrm{S}}^{\mathrm{TZ}}$ values from global tomography, on the other. The resolution of global wavespeed heterogeneity in the $\mathrm{TZ}$ is most uniform $(30,31)$ at wavelengths that are much larger $(>3000 \mathrm{~km})$ than the spatial resolution of the $t_{\text {diff }}$ measurements $(\approx 500$ $\mathrm{km}$ ), and this may obscure existing $t_{\text {diff }}-V_{\mathrm{S}}^{\mathrm{TZ}}$ correlations. Our study, in which $t_{\text {diff }}$ and $V_{\mathrm{S}}^{\mathrm{TZ}}$ relate to the same spatial length scale, corroborates models in which the phase transformations in olivine cause both 410 and 660 .

\section{References and Notes}

1. J. D. Bernal, Observatory 59, 265 (1936).

2. D. L. Anderson, Science 157, 1165 (1967).

3. A. E. Ringwood, Earth Planet. Sci. Lett. 5, 401 (1969).

4. E. Ito, E. Takahashi, J. Geophys. Res. 94, 10673 (1989).

5. T. Katsura, E. Ito, J. Geophys. Res. 94, 15663 (1989).

6. G. Helffrich, Rev. Geophys. 38, 141 (2000).

7. P. M. Shearer, in Earth's Deep Interior: Mineral Physics and Tomography from the Atomic to the Global Scale, S. Karato, A. M. Forte, R. C. Liebermann, G. Masters, L. Stixrude, Eds. (Geophysical Monograph 117, American Geophysical Union, Washington, DC, 2000), pp. 115-131.

8. S.-H. Shim, T. S. Duffy, G. Shen, Nature 411, 571 (2001).

9. L. Chudinovskikh, R. Boehler, Nature 411, 574 (2001).

10. T. Irifune et al., Science 279, 1698 (1998).

11. K. Hirose, Y. Fei, S. Ono, T. Yagi, K. Funakoshi, Earth Planet. Sci. Lett. 184, 567 (2001).

12. S. Chevrot, L. Vinnik, J.-P. Montagner, J. Geophys. Res. 104, 20203 (1999).

13. M. P. Flanagan, P. M. Shearer, J. Geophys. Res. 103 , 2673 (1998).

14. C. R. Bina, G. R. Helffrich, J. Geophys. Res. 99, 15853 (1994).

15. Because phase transformations in the Earth occur over depth intervals in which multiple phases coexist, it is not meaningful to define the Clapeyron slopes at 410 or 660 thermodynamically (14). The discontinuities, however, are seen as sharp interfaces by finite-frequency seismic waves, and measurements of the depths to these apparent interfaces can constrain the effective ("seismic") Clapeyron slopes at the discontinuities which, although weakly dependent on seismic-wave frequency, otherwise are determined only by thermodynamic properties
(14). The seismically derived Clapeyron slopes can also be affected by isostructural phases with variable chemistry at the depth of the discontinuities (32), in particular because of the exchange of magnesium and iron between olivine and other mantle minerals $(33,34)$. According to the phase diagrams from (5), a $1 \%$ increase in the magnesium number $\mathrm{Mg} \#=\mathrm{Mg} /(\mathrm{Mg}+\mathrm{Fe})$ would raise the pressure of $\alpha \rightarrow \beta$ by $0.1 \mathrm{GPa}$ (increasing the depth to 410 by $\delta d_{410} \approx 3 \mathrm{~km}$ ), the same effect as from a $35 \mathrm{~K}$ increase in temperature [given $\gamma_{410}=2.9 \mathrm{MPa} / \mathrm{K}$ (14)]. With $P$ and $S$ velocities $\left(V_{P, S}\right)$ also growing with $\mathrm{Mg \#} \mathrm{(33),} \mathrm{lateral} \mathrm{variations} \mathrm{in} \mathrm{Mg \#} \mathrm{would} \mathrm{thus} \mathrm{weaken}$ the thermally induced anticorrelation between $d_{410}$ and $V_{\mathrm{P}, \mathrm{S}}$ depicted in Fig. 1A. Our observation that the "seismic" Clapeyron slopes agree (within uncertainties) with the mineralogical slopes for $\mathrm{Mg}_{2} \mathrm{SiO}_{4}$ is consistent with the actual effect of composition being small.

16. D. J. Weidner, Y. Wang, in Earth's Deep Interior: Mineral Physics and Tomography from the Atomic to the Global Scale, S. Karato, A. M. Forte, R. C. Liebermann, G. Masters, L. Stixrude, Eds. (Geophysical Monograph 117, American Geophysical Union, Washington, DC, 2000), pp. 215-235.

17. A downwarp of 660 and an uplift of 410 have been documented beneath the "cold" subduction zones ( 35 , 36), but these regions represent only a small fraction of the upper mantle, possibly with compositional and kinetic effects affecting the phase transformations (16). At larger scales, there is evidence both for and against a $\delta d_{410}-\delta d_{660}$ anticorrelation $(6,7)$, but this may be inconclusive owing to the possibly incoherent temperature variations in the upper and lower TZ (13) and to uncertainties of the measurements (errors in the models of the heterogeneous mantle above 410 translate into substantial uncertainties in inferred discontinuity depth). The measurements of $H_{\mathrm{TZ}}$ are generally more precise than those of $\delta d_{410,60}$, because $H_{\mathrm{TZ}}$ can be constrained with observables that to first order do not depend on structure above 410 , such as the $t_{\text {diff }}=t_{P 660 s}-t_{P 410 s}$ that we use here. However, a recent global compilation of converted-wave differential times $t_{\text {diff }}=t_{P 6605}-t_{P 4105}$ (which scale with $H_{\mathrm{TZ}}$ ) showed poor correlation with seismic velocities from global tomographic models (12). A similarly weak correlation was obtained by mapping $H_{\mathrm{TZ}}$ with long-period SS precursors (13).

18. S. Lebedev, G. Nolet, in preparation.

19. F. J. Simons, A. Zielhuis, R. D. van der Hilst, Lithos $\mathbf{4 8}$, 17 (1999).

20. L. P. Vinnik, Phys. Earth Planet. Int. 15, 39 (1977).

21. B. Efron, R. Tibshirani, Science 253, 390 (1991).

22. For each station, we collected all available traces and inspected them visually, rejecting those with strong background noise before the $P$-wave onset. After the rotation and deconvolution, we also discarded traces with the SV-component amplitude exceeding $15 \%$ of that of the $P$ wave. Finally, we rejected the data if $P 410$ s or $P 660$ s arrivals could not be identified on the stacks (presumably due to multipathing caused by strong upper-mantle heterogeneity or small-scale discontinuity undulations). The number of records that contribute to the stacks ranges from 13 to 135 , with an average of 53 (37).

23. We stack the records from events at different azimuths and distances so that the piercing points of $P \mathrm{~d} s$ waves at the discontinuities are distributed over an area a few hundred kilometers wide (Fig. 1B). Taking into account the $100-$ to $300-\mathrm{km}$ width of the Fresnel zone of the waves at the discontinuities [see (12) for discussion], we estimate the lateral resolution of the measurements at $\approx 500 \mathrm{~km}$.

24. Supplementary Web material is available on Science Online at www.sciencemag.org/cgi/content/full/296/ 5571/1300/DC1.

25. B. L. N. Kennett, E. R. Engdahl, Geophys. J. Int. 105, 429 (1991).

26. A. M. Dziewonski, D. L. Anderson, Phys. Earth Planet. Int. 25, 297 (1981).

27. S. Karato, Geophys. Res. Lett. 20, 1623 (1993).

28. W. H. Press, S. A. Teukolsky, W. T. Vetterling, B. P. Flannery, Numerical Recipes in FORTRAN (Cambridge Univ. Press, New York, ed. 2, 1992).

29. The correlation of global maps of $H_{\mathrm{TZ}}$ obtained using SS precursors with tomographic TZ wavespeeds is weak (13), although consistent with the olivine Clapeyron slopes from (14).

30. Reference Earth Model Web site, http://mahi.ucsd. edu/Gabi/rem.html.

31. T. W. Becker, L. Boschi, Geochem. Geophys. Geosyst.

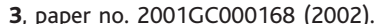

32. L. Stixrude, J. Geophys. Res. 102, 14835 (1997).

33. T. Irifune, M. Isshiki, Nature 392, 702 (1998).

34. C. Bina, Nature 392, 650 (1998).

35. J. E. Vidale, H. M. Benz, Nature 356, 678 (1992)

36. C. W. Wicks, M. A. Richards, Science 261, 1424 (1993).

37. S. Lebedev, S. Chevrot, R. D. van der Hilst, in preparation.

38. G. Nolet, J. Geophys. Res. 95, 8499 (1990).

39. We thank A. Zielhuis for running the resolution tests for the tomographic model of Australia. This work was supported by the David and Lucile Packard Foundation through a fellowship awarded to R.D.v.d.H.

27 December 2001; accepted 29 March 2002

\title{
Identity and Search in Social Networks
}

\author{
Duncan J. Watts, ${ }^{1,2,3 *}$ Peter Sheridan Dodds, ${ }^{2}$ M. E. J. Newman ${ }^{3}$
}

\begin{abstract}
Social networks have the surprising property of being "searchable": Ordinary people are capable of directing messages through their network of acquaintances to reach a specific but distant target person in only a few steps. We present a model that offers an explanation of social network searchability in terms of recognizable personal identities: sets of characteristics measured along a number of social dimensions. Our model defines a class of searchable networks and a method for searching them that may be applicable to many network search problems, including the location of data files in peer-to-peer networks, pages on the World Wide Web, and information in distributed databases.
\end{abstract}

In the late 1960s, Travers and Milgram (1) conducted an experiment in which randomly selected individuals in Boston, Massachusetts, and Omaha, Nebraska, were asked to direct letters to a target person in Boston, each forwarding his or her letter to a single acquaintance whom they judged to be closer than themselves to the target. Subsequent recipients did the same. The average length of the resulting acquaintance chains for the let- 